\title{
Statistics of Joule heating in the auroral zone and polar cap using Astrid-2 satellite Poynting flux
}

\author{
A. Olsson ${ }^{1}$, P. Janhunen ${ }^{2}$, T. Karlsson ${ }^{3}$, N. Ivchenko ${ }^{3}$, and L. G. Blomberg ${ }^{3}$ \\ ${ }^{1}$ Swedish Institute of Space Physics, Uppsala, Sweden \\ ${ }^{2}$ Finnish Meteorological Institute, Space Research, Helsinki, Finland \\ ${ }^{3}$ Alfvén Laboratory, Royal Institute of Techology, Stockholm, Sweden
}

Received: 2 July 2004 - Revised: 20 September 2004 - Accepted: 5 October 2004 - Published: 22 December 2004

\begin{abstract}
We make a statistical study of ionospheric Joule heating with the Poynting flux method using six months of Astrid-2/EMMA electric and magnetic field data during 1999 (solar maximum year). For the background magnetic field we use the IGRF model. Our results are in agreement with earlier statistical satellite studies using both the $\Sigma_{P} E^{2}$ method and the Poynting flux method. We present a rather comprehensive set of fitted Joule heating formulas expressing the Joule heating in given magnetic local time (MLT) and invariant latitude (ILAT) range under given solar illumination conditions as a function of the $K_{p}$ index, the $A E$ index, the Akasofu epsilon parameter and the solar wind kinetic energy flux. The study thus provides improved and more detailed estimates of the statistical Joule heating. Such estimates are necessary building blocks for future quantitative studies of the power budget in the magnetosphere and in the nightside auroral region.
\end{abstract}

Key words. Ionosphere (electric fields and currents; ionosphere-magnetosphere interactions) - Magnetospheric physics (magnetospheric configuration and dynamics)

\section{Introduction}

Joule heating $(\mathrm{JH})$ or frictional heating is a high latitude ionospheric phenomenon where the drift energy of ionospheric ions turns into thermal and bulk kinetic energy of neutrals due to collisions (Richmond, 1995). The $\mathrm{JH}\left(\mathrm{W} \mathrm{m}^{-2}\right)$ is calculated from the product of the electric field and the heightintegrated current density, E.J. Alternatively, if there is no neutral wind, it can be expressed as the product of the heightintegrated Pedersen conductivity and the square of the electric field, $\Sigma_{P} E^{2}$. Global JH has been studied exprimentally by radar (Vickrey et al., 1982) and low-orbiting satellite (Foster et al., 1983). In the latter case, the E-field is measured directly and $\Sigma_{P}$ is computed from a model, using the measured

Correspondence to: A. Olsson

(ao@irfu.se) precipitating particle fluxes as input. The neutral wind is taken from a model or assumed to be zero.

Another way to obtain the JH (E.J) is to apply the Poynting theorem and estimate the field-aligned Poynting flux $\left(S_{\|}=\hat{b} \cdot \mathbf{E} \times \mathbf{B}_{1} / \mu_{0}\right)$, where $\mathbf{B}_{1}=\mathbf{B}-\mathbf{B}_{0}$ and $\mathbf{B}_{0}$ is the background magnetic field (Kelley et al., 1991). In earlier studies using this approach (Gary et al., 1994, 1995), $\mathbf{B}_{0}$ was determined from those portions of the data that were outside the auroral zone.

Already in the eighties, Foster et al. (1983) and Ahn et al. (1983) statistically gave the hemispheric JH as a function of the $K_{p}$ and $A E / \mathrm{AL}$ indices. Foster et al. (1983) used AE-C satellite data during four years of solar minimum. This comprehensive study (25000 orbits) used the $\Sigma_{P} E^{2}$ method, with neutral wind assumed to be zero. They needed a model of the conductivity depending on precipitating auroral electrons, as well as the the solar EUV. It was shown how the hemispheric $\mathrm{JH}$ in GW increases with the $K_{p}$ index as $\mathrm{JH}=4+20 K_{p}$. The invariant latitude (ILAT) range considered was 55-85. Ahn et al. (1983) reported results of JH rate as a function of the geomagnetic indices $A E$ and $\mathrm{AL}$ from Chatanika radar data using three days of data.

The satellite Poynting flux method was employed by Gary et al. (1994) to study JH statistically, using 576 DE-2 passes during solar maximum. The study covered dawn-dusk predominantly in summer/winter and noon-midnight passes near the equinoxes.

Being an important channel in the magnetospheric total power balance (see, e.g. Tanskanen et al., 2002, for a recent discussion), ionospheric $\mathrm{JH}$ has always been an important question. One of the reasons that warrants interest in this topic right now, however, is that recently, ionospheric power input (JH and particle precipitation) from a global MHD simulation was calculated by Palmroth et al. (2004). The JH power as found from the simulation was much smaller than what is given by the $A E$-index formula of Ahn et al. (1983). Since the same simulation produces a cross-polar potential drop which is in good agreement with SuperDARN radar measurements (Janhunen et al., 1998), it is not self-evident 
that the simulated JH level is wrong. Other MHD simulations may produce larger JH levels, but they also clearly overestimate the cross-polar potential drop. The cross-polar potential drop has also been calculated from the same Astrid-2/EMMA database than what is used in this study and was found to be in good agreement with other studies (Eriksson et al., 2001).

In this paper we estimate the statistical $\mathrm{JH}$ rate from the Poynting flux formula using 6 months of Astrid-2/EMMA low-orbiting satellite data. An advantage with this method is that no models for the conductivity or neutral wind effects are needed. The purpose of the paper is not only to present a new JH data set and compare it with previously published results, but also to express the JH in different regions, defined by magnetic local time (MLT) and ILAT, and different conditions as a function of different index $\left(K_{p}\right.$ and $\left.A E\right)$ and solar wind parameters ( $\epsilon$ and $W$ defined by Eqs. (8) and (9) below), thus giving a more complete picture than what a single statistical formula would give.

The structure of the paper is as follows. We first present the instrumentation and data analysis. We then consider statistically the global $\mathrm{JH}$ and $\mathrm{JH}$ in the nominal nightside auroral zone only (65-74 MLT, 18-06 ILAT; also decomposed into three MLT sectors 18-22, 22-02, 22-06). In both cases the results are binned for $K_{p}$ and $A E$ indices. Global and auroral $\mathrm{JH}$ as a function of solar wind parameters are considered thereafter. The paper ends with summary and discussion sections.

\section{Theory}

The Poynting theorem is derived by computing the time rate of change in the electromagnetic energy density and using Maxwell's equations:

$$
\begin{aligned}
& \frac{\partial}{\partial t}\left(\frac{1}{2} \epsilon_{0} E^{2}+\frac{1}{2 \mu_{0}} B^{2}\right) \\
= & \epsilon_{0} \mathbf{E} \cdot \frac{\partial \mathbf{E}}{\partial t}+\frac{1}{\mu_{0}} \mathbf{B} \cdot \frac{\partial \mathbf{B}}{\partial t} \\
= & \frac{1}{\mu_{0}} \mathbf{E} \cdot \nabla \times \mathbf{B}-\mathbf{j} \cdot \mathbf{E}-\frac{1}{\mu_{0}} \mathbf{B} \cdot \nabla \times \mathbf{E} \\
= & -\frac{1}{\mu_{0}} \nabla \cdot(\mathbf{E} \times \mathbf{B})-\mathbf{j} \cdot \mathbf{E} \\
\equiv & -\nabla \cdot \mathbf{S}-\mathbf{j} \cdot \mathbf{E} .
\end{aligned}
$$

Taking a time average, or a statistical ensemble average, the time derivative terms disappear. Integrating the result over a volume $V$ and using Gauss' theorem one obtains for the consumed JH

$\mathrm{JH}=\int_{V} d V \mathbf{j} \cdot \mathbf{E}=-\int_{V} d V \nabla \cdot \mathbf{S}=-\oint_{\partial V} d \mathbf{a} \cdot \mathbf{S}$,

where $d \mathbf{a}$ is an outward surface element.

Writing $\mathbf{B}=\mathbf{B}_{0}+\mathbf{B}_{1}$, where $\mathbf{B}_{0}$ is the curl-free background field, we obtain (Kelley et al., 1991)

$\mu_{0} \mathrm{JH}=-\int_{V} d V \nabla \cdot\left(\mathbf{E} \times \mathbf{B}_{0}\right)-\oint_{\partial V} d \mathbf{a} \cdot \mathbf{E} \times \mathbf{B}_{1}$.
The first term vanishes when a time or ensemble average is taken:

$\nabla \cdot\left(\mathbf{E} \times \mathbf{B}_{0}\right)=(\nabla \times \mathbf{E}) \cdot \mathbf{B}_{0}-\left(\nabla \times \mathbf{B}_{0}\right) \cdot \mathbf{E}$,

where $\nabla \times \mathbf{E}=-\partial \mathbf{B}_{1} / \partial t \rightarrow 0$ and $\nabla \times \mathbf{B}_{0}=0$ by assumption. Thus, only the second term contributes to JH. In that term, $\mathbf{E}$ is (physically and by data analysis construction) perpendicular to $\mathbf{B}$ and thus nearly perpendicular to $\mathbf{B}_{0}$ since $\left|\mathbf{B}_{1}\right| \ll\left|\mathbf{B}_{0}\right|$. Furthermore, $\mathbf{B}_{1}$ is also almost perpendicular to $\mathbf{B}_{0}$ since $\mathbf{B}_{1}$ is mainly created by local currents which must be nearly field-aligned in the low-beta plasma existing at $1000 \mathrm{~km}$ altitude. Thus, the cross product $\mathbf{E} \times \mathbf{B}_{1}$ is nearly parallel to $\mathbf{B}_{0}$ and replacing it by the parallel component is a good approximation:

$\mu_{0} \mathrm{JH} \approx-\oint_{\partial V} d \mathbf{a} \cdot \hat{\boldsymbol{b}_{0}} \hat{\boldsymbol{b}_{0}} \cdot \mathbf{E} \times \mathbf{B}_{1}$.

Here $\hat{\boldsymbol{b}_{0}}$ is unit vector along $\mathbf{B}_{0}$. We have also verified the goodness of this approximation using our data.

In principle, the integration volume $V$ includes the whole Earth. Knowing that there are intense dynamo currents in the liquid core, the first term of Eq. (3) does not necessarily vanish there. However, Poynting flux arriving from space cannot penetrate the core region because it is surrounded by a highly conducting mantle that forces $\mathbf{E} \approx 0$ and thus $\mathbf{S} \approx 0$ in the mantle region. So, the first term indeed vanishes.

The quantity $\mathrm{JH}$ thus represents $\mathbf{j} \cdot \mathbf{E}$ integrated over the ionosphere, atmosphere and upper crust. In the atmosphere, no JH occurs, but in the upper crust, induction causes Earth currents to flow. Let us estimate the contribution of the Earth current JH relative to ionospheric JH. Starting from Eq. (5.169) of Jackson (1999), one can derive for their ratio the expression $(1 / 4)\left(\Sigma_{H} / \Sigma_{P}\right)\left(\Sigma_{H} /\left(\sigma_{0} \delta\right)\right)$, where $\Sigma_{P, H}$ is the ionospheric height-integrated Pedersen and Hall conductivity, respectively, $\sigma_{0}$ is the Earth conductivity, $\delta=\sqrt{2 /\left(\mu_{0} \sigma_{0} \omega\right)}$ the skin depth and $\omega$ the angular frequency of variations. For $\Sigma_{P}=20 \Omega^{-1}, \Sigma_{H}=60 \Omega^{-1}$, $\sigma_{0}=10^{-2} \Omega^{-1} \mathrm{~m}^{-1}, \omega=2 \pi /(60 \mathrm{~s}), \delta=39 \mathrm{~km}$ one obtains that $12 \%$ of power is consumed in the crust and $88 \%$ in the ionosphere. Since the selected numerical values represent a rather extreme case, the Earth current contribution to JH is usually negligible and our $\mathrm{JH}$ represents mainly the ionospheric $\mathrm{JH}$.

Using vector identities, one can write $\hat{\boldsymbol{b}_{0}} \cdot \mathbf{E} \times \mathbf{B}$ in many equivalent and some numerically nearly equivalent ways, e.g.:

$\hat{\boldsymbol{b}_{0}} \cdot \mathbf{E} \times \mathbf{B}=\hat{\boldsymbol{b}_{0}} \cdot \mathbf{E} \times \mathbf{B}_{1}=\frac{B}{B_{0}} \hat{\mathbf{b}} \cdot \mathbf{E} \times \mathbf{B}_{1}$,

where $B / B_{0} \approx 1$ is a good approximation at low altitude. These alternative forms appear in some of the literature.

\section{Instrumentation and data analysis}

The Astrid-2 satellite was launched in late December 1998 and was orbiting in the ionosphere at apogee around $1000 \mathrm{~km}$, producing about 6 months of data and completing 
about 3000 nearly polar orbits (Marklund et al., 2001). We use the DC electric and magnetic field instrument (EMMA, Blomberg et al., 2004) to compute JH using the Poynting flux method. The full time resolution is at least 16 samples per second; in this study, however, we use 1-s averaged data for both $\mathbf{E}$ and $\mathbf{B}$. As a complement to the previous large statistical study (Foster et al., 1983), we obtain the statistics for solar maximum conditions. We will not only study the $K_{p}$ and seasonal dependence on the $\mathrm{JH}$, but also present a more detailed study of the MLT dependence and ILAT.

The EMMA instrument measures the full 3-D magnetic field and two components of the electric field in the spin plane. The electric field is originally given in an inertial (GEI) coordinate system. Before using it we transform it to a corotating frame to eliminate the corotation electric field contribution. The spin axis is nominally oriented towards the Sun. The full 3-D electric field is computed by assuming $\mathbf{E} \cdot \mathbf{B}=0$. When the $\mathbf{B}$-field is nearly in the spin plane, this method would break down. We therefore use only those data for which the angle between the magnetic field and the spin plane is at least $15^{\circ}$ (Ivchenko et al., 2001). From the $\mathbf{E}$ and B fields, we compute the downward Poynting flux as

$S_{\text {down }}=s \frac{1}{\mu_{0}} \hat{\boldsymbol{b}_{0}} \cdot \mathbf{E} \times \mathbf{B}\left(\frac{B_{i}}{B}\right) \cos I$

where $s=+1$ and $s=-1$ in the Northern and Southern Hemisphere, respectively, $\hat{\boldsymbol{b}_{0}}$ is a unit vector in the direction of $\mathbf{B}_{0}$, where $\mathbf{B}_{0}$ is the model background magnetic field. For $\mathbf{B}_{0}$, we use the IGRF model. The factor $B_{i} / B$ takes into account Poynting flux convergence due to flux tube scaling from the satellite altitude $(1000 \mathrm{~km})$ down to the ionosphere $(110 \mathrm{~km})$. Finally, $\cos I$, where $I$ is the inclination angle of the magnetic field at the satellite footpoint (sharp angle between $\mathbf{B}_{0}$ and vertical), takes care of obtaining the right amount of power per unit area in the ionosphere.

The total number of Southern and Northern Hemisphere high-latitude traversals having $\mathbf{E}$ and $\mathbf{B}$ field data is 4034 . For each crossing we generated a plot displaying the raw measured field components, as well as the calculated $S_{\text {down }}$. Each plot was visually inspected to ensure that the field measurements did not contain data errors. An example of such a plot in showed in Fig. 1. A total of 2690 crossings were fully acceptable, 1196 contained parts that were excluded and 148 were completely rejected. The total length of the accepted data is $675 \mathrm{~h}$ ( 2.43 million points).

\section{Statistical global JH}

For the statistical global JH, data are included from all MLT sectors and for ILAT values covering both the polar cap regions and the auroral zone. The regions where the most significant $\mathrm{JH}$ takes place is in the ILAT range 55-90. In this ILAT range we will be able to compare our results with those of Foster et al. (1983) and Ahn et al. (1983). In the next section we specifically study the $\mathrm{JH}$ in the nightside auroral zone

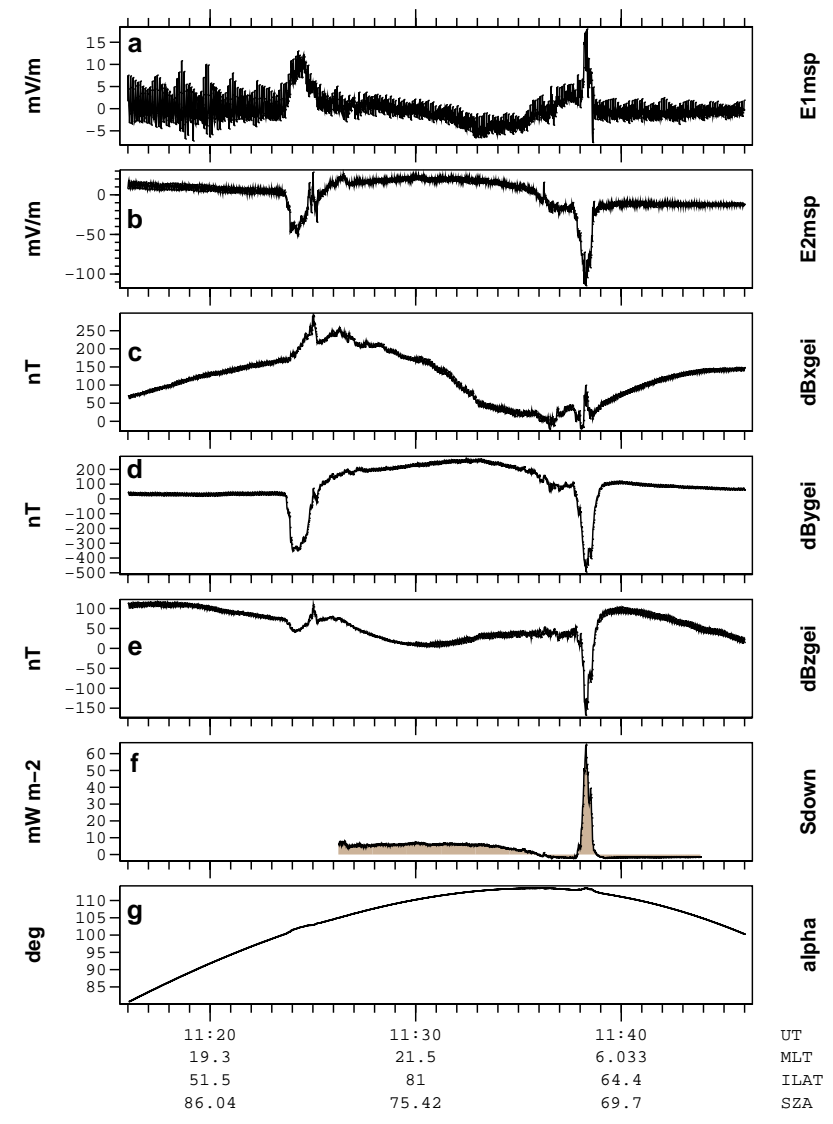

Fig. 1. Example plot used in visual inspection of events. (a-b) spin plane electric field in msp coordinates, (c-e) IGRF-subtracted magnetic field in GEI coordinates, (f) calculated downward fieldaligned Poynting flux, (g) angle $\alpha$ between spin axis and magnetic field, Poynting fluxes for $75^{\circ}<\alpha<105^{\circ}$ were not shown in panel (f).

(we define "nightside" to mean 18-06 MLT, regardless of the position of the terminator).

In Fig. 2 the average global JH is shown for $K_{p} \leq 2$ (ILAT 55-90). Nightside JH correlates rather well with the auroral zone. Significant JH is also seen in the cusp region. In the right plot, the corresponding orbital coverage (number of high-altitude traversals contributing to each bin) is shown. The orbital coverage is good in all regions but the highest ILAT ( $>85$ or so). Negative downward Poynting flux values that occur in a few individual bins are displayed as zero.

Figure 3 is similar in format to Fig. 2 but for $K_{p}>2$. The $\mathrm{JH}$ is more intense than for low $K_{p}$ and spread over a larger ILAT range. Significant JH now also occurs near 18 and 06 MLT. Overall, the pattern is more irregular than for low $K_{p}$, which is not surprising.

In Fig. 4 a line plot shows how the total global downward Poynting flux varies in the ILAT range $40-90^{\circ}$. The flux goes smoothly to zero at about ILAT 55, as it should since we calculate the Poynting flux in a reference frame that rotates with the Earth. Formulas for the global JH in different solar illumination conditions are found in Table 1, row 1-3. 

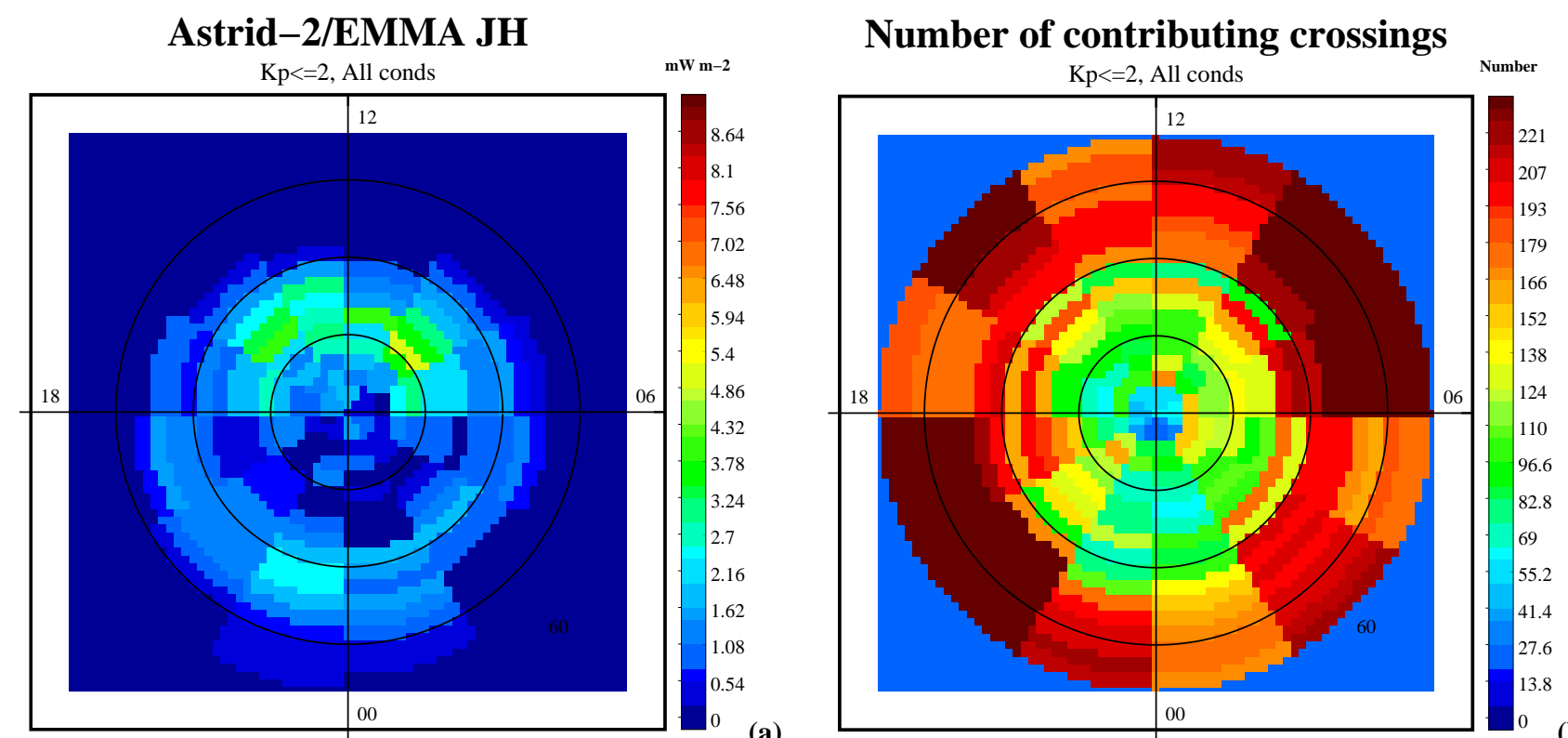

(a)

(b)

Fig. 2. The average Joule heating for ILAT $55-90$ for $K_{p} \leq 2$, all MLT (left). MLT 12, 18, 24 and 06 are shown in the plot. The inner circle corresponds to ILAT 80 . The Joule heating is correlated with the auroral zone and in the dayside the intense Joule heating is related to the cusp region. In the right subplot the corresponding orbital coverage (number of auroral crossings contributing to each bin) is shown.
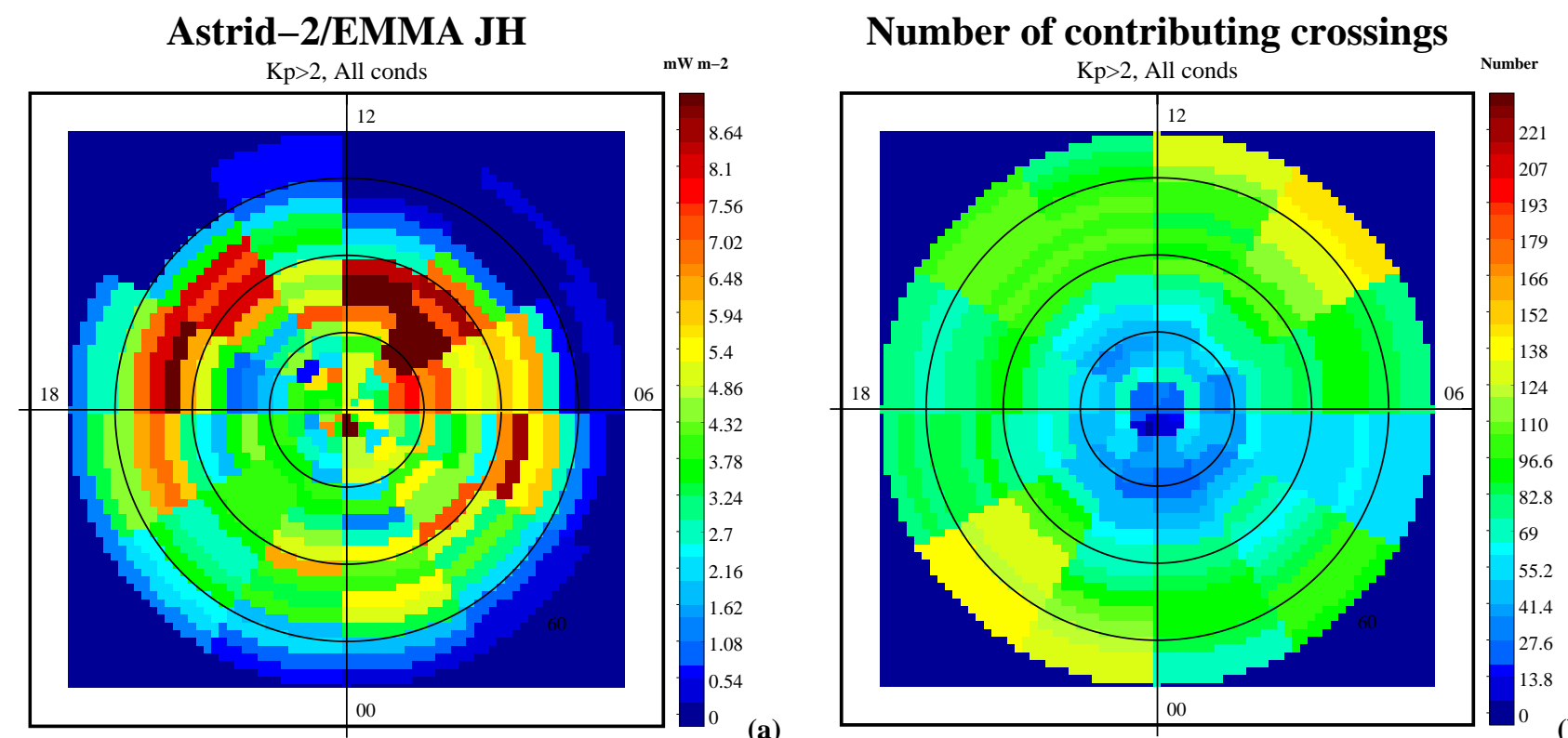

(a)

(b)

Fig. 3. Same as Fig. 2 but for $K_{p}>2$. The average Joule heating is overall higher, often by a factor of 2 and is more significant in wider ILAT range.

To be able to compare the results from Astrid-2/EMMA data with those from previous satellite and radar measurements (Foster et al., 1983) and (Ahn et al., 1983), we again study the global average $\mathrm{JH}$ in the ILAT range 55-90. In Fig. 5 it is shown how the JH varies with $K_{p}$ for different solar illumination conditions of the satellite footpoint. The JH (in GW) estimated from Astrid-2/EMMA Poynting flux is shown as dots. The error bars in Fig. 5 and elsewhere correspond to partitioning the data set randomly in two halves and computing the value separately for each. A linear fit to the data is shown by a solid line. The formula for the fit is seen in the plots, as well as in Table 1, rows 4-6. In the fits we used only the four lowest data points because beyond those the error bars become much larger in most cases. The 
Table 1. Joule heating $(\mathrm{GW})$ as a function of $K_{p}$ index in different hemispheric regions.

\begin{tabular}{llllll}
\hline & All MLT & MLT 18-06 & MLT 18-22 & MLT 22-02 & MLT 02-06 \\
\hline ILAT 55-90, All conds & $30 K_{p}-7.6$ & & & & \\
ILAT 55-90, Sunlit & $27 K_{p}$ & & & & \\
ILAT 55-90, Darkness & $24 K_{p}-12$ & & & & \\
\hline ILAT 65-74, All conds & & $6.8 K_{p}+0.8$ & $1.9 K_{p}+0.7$ & $2.3 K_{p}+1$ & $2.6 K_{p}-1$ \\
ILAT 65-74, Sunlit & & $6.5 K_{p}+0.5$ & $2.3 K_{p}-1$ & $2.2 K_{p}+1.3$ & $2 K_{p}+0.3$ \\
ILAT 65-74, Darkness & & $8.5 K_{p}-2$ & $1.7 K_{p}+2$ & $2.2 K_{p}+0.8$ & - \\
\hline
\end{tabular}

Table 2. Joule heating (GW) as a function of $A E$ index (nT) in different hemispheric regions.

\begin{tabular}{llllll}
\hline & All MLT & MLT 18-06 & MLT 18-22 & MLT 22-02 & MLT 02-06 \\
\hline ILAT 55-90, All conds & $0.36 A E$ & & & & \\
ILAT 55-90, Sunlit & $0.37 A E$ & & & & \\
ILAT 55-90, Darkness & $0.24 A E-7$ & & & & \\
\hline ILAT 65-74, All conds & & $0.07 A E+4$ & $0.03 A E$ & $0.017 A E+3.6$ & $0.025 A E$ \\
ILAT 65-74, Sunlit & & $0.068 A E+4$ & $0.026 A E$ & $0.017 A E+3.5$ & $0.024 A E$ \\
ILAT 65-74, Darkness & & $0.08 A E+1$ & $0.03 A E$ & $0.013 A E+4$ & $0.04 A E-3.6$ \\
\hline
\end{tabular}

dashed line is the JH rate estimated by Foster et al. (1983) for ILAT 55-85 $\left(\mathrm{JH}=\left(20 K_{p}+4\right) \mathrm{GW}\right)$. The JH estimated from EMMA data is for $K_{p}>2$ somewhat but not much higher than the JH estimated of Foster et al. (1983). The global JH is about twice as large for sunlit conditions compared to darkness, which points to the fact that $\mathrm{JH}$ due to the cusp region is a significant source. Estimates for the total global $\mathrm{JH}$ for different solar illumination, and low $K_{p}\left(0 \leq K_{p} \leq 2\right)$ and high $K_{p}\left(K_{p}>2\right)$, can be found in Table 4, column 1 .

The data shown in Fig. 5 and in other similar plots to follow was processed so that the average JH level was first computed for each ILAT and MLT bin separately and then the total $\mathrm{JH}$ was summed from those bins by weighting with the bin area. The ILAT bin size is two degrees and the MLT binning two hours. An alternative method would be to treat the entire desired MLT-ILAT range as one bin. Using this alternative method, the statistical scatter could be reduced, but at the expense of not taking properly into account the nonuniform orbital coverage.

In Fig. 6 the same parameters are used as in Fig. 5 but now for $A E$. In the left subplot we see that the JH estimated from EMMA data is somewhat higher than the fitted formula of Ahn et al. (1983) (JH/GW=0.23 $A E / n T$, dotted line). As was the case for the $K_{p}$, we can see that the $\mathrm{JH}$ is higher during sunlit conditions compared to darkness. It is interesting to notice that for $A E>400$, the $\mathrm{JH}$ shows a tendency to saturate, or, in other words, if $A E$ is considered as a function of the $\mathrm{JH}, A E$ grows faster than linearly for high values of the JH. The explanation may be that not only the mean value but also the spatial variability of the strength of horizontal ionospheric currents likely increases when the activity level

\section{MLT-averaged Poynting flux}

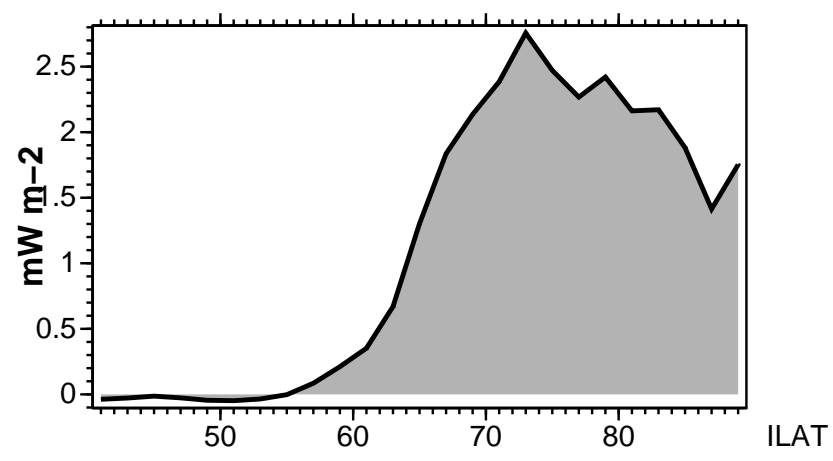

Fig. 4. Average global (ILAT 25-90) Joule heating for all $K_{p}$ values and all MLT sectors. At latitudes above ILAT 60 we have an average downward Poynting flux.

(measured by $K_{p}$ or the $\mathrm{JH}$ ) increases, and thus $A E$, being defined as the maximum magnetic perturbation over a set of stations, reacts superlinearly. The solid line represents a fit to the data and the formula for this is seen in each plot, as well as in Table 2, rows 4-6. Estimates for the total global $\mathrm{JH}$ for different solar illumination can be found in Table 4, column 1. 
Astrid-2/EMMA JH, All conds

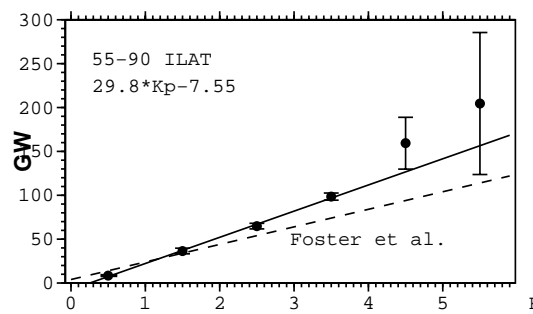

Astrid-2/EMMA JH, Sunlit

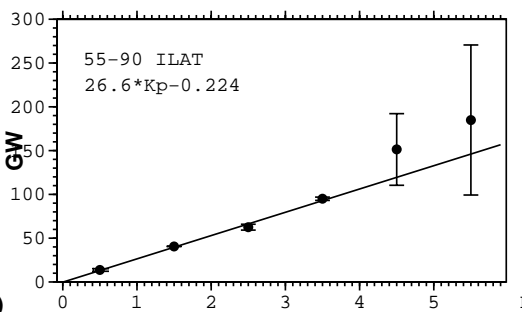

Astrid-2/EMMA JH, Darkness

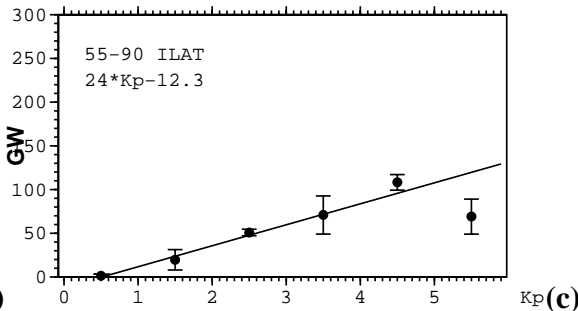

Fig. 5. The average Joule heating dependence on $K_{p}$ for ILAT 55-90, all MLT and for (a) all solar illumination conditions, (b) sunlit conditions and (c) darkness conditions. In each subplot, the solid line represents a fit to the data and the corresponding formula is also shown (see also Table 1, row 4-6). The error bars correspond to partitioning the data set randomly into two halves and computing the value separately for each. The Joule heating is for all $K_{p}$ somewhat higher during sunlit conditions. In subplot (a) comparisons with the results of Foster et al. (1983) give that the Astrid-2/EMMA study indicates higher estimates of the Joule heating for $K_{p}>2$.

Astrid-2/EMMA JH, All conds

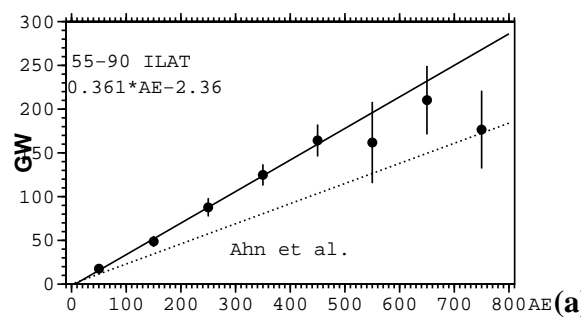

Astrid-2/EMMA JH, Sunlit

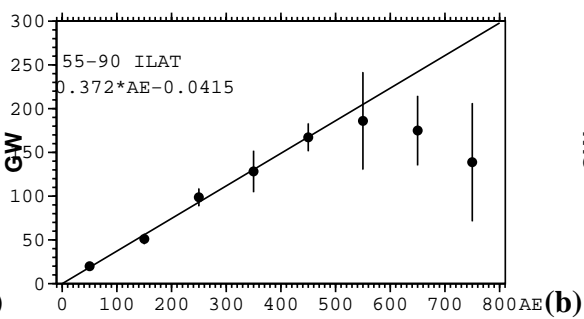

Astrid-2/EMMA JH, Darkness

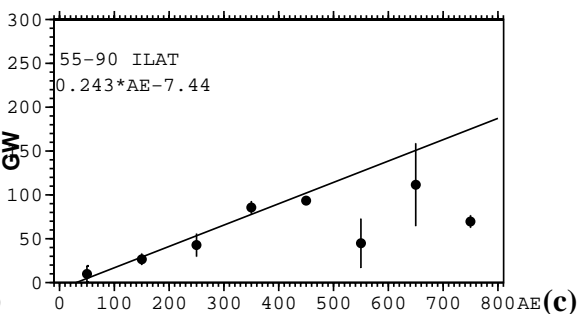

Fig. 6. Same as Fig. 5 but for $A E$ indexes. The Joule heating is almost twice as high for sunlit conditions compared to darkness. At $A E$ values above $\sim 400$ an interesting saturation of the Joule heating occurrs. In subplot (a) comparisons with the results of Ahn et al. (1983) (dotted line) is shown. For all $K_{p}$ the Joule heating estimates from Astrid-2/EMMA are always higher than the radar estimates by Ahn et al. (1983).

Astrid-2/EMMA JH, All conds

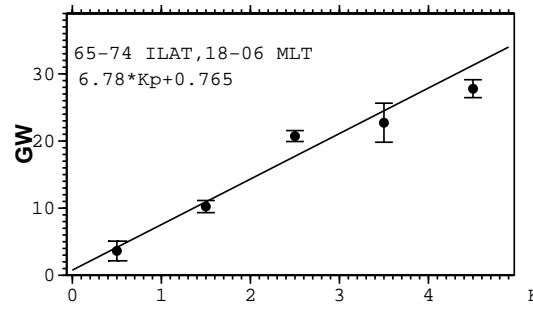

Astrid-2/EMMA JH, Sunlit

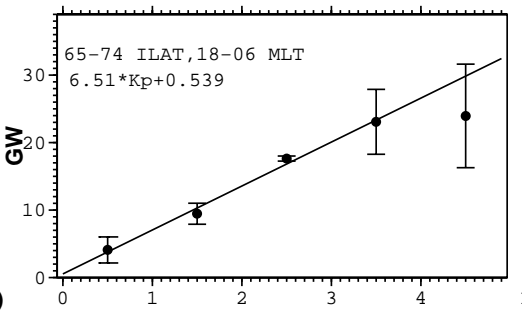

Astrid-2/EMMA JH, Darkness

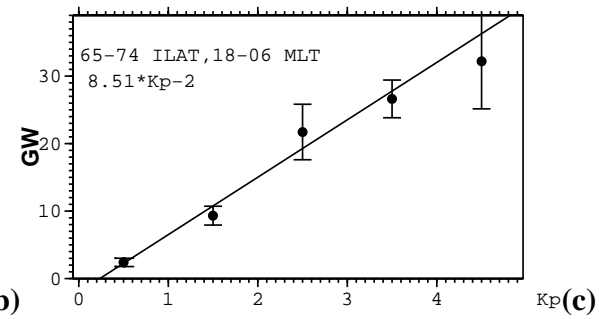

Fig. 7. The average Joule heating for the nightside (MLT 18-06) auroral zone (ILAT 65-74) for (a) all solar illumination conditions, (b) sunlit conditions and (c) darkness conditions. The solid line shows a fit to the data and the formula for the Joule heating is shown in each subplot (see also Table 1, row 7-9). The Joule heating is larger for darkness conditions especially for $K_{p}>2$.

\section{Statistical JH in nightside auroral zone}

In this section we study the statistical estimates of the average $\mathrm{JH}$ in the nightside (MLT 18-06) nominal auroral zone (ILAT 65-74).

In Fig. 7 the dependence of JH on $K_{p}$ is shown for different solar illumination conditions. A fit to the data is shown by the solid line and the corresponding formula is shown in each subplot, as well as in Table 1, rows 4-6. Estimates of the total JH can be found in Table 4, column 2. The JH in the nightside auroral zone is about $20-30 \%$ of the total global $\mathrm{JH}$ (ratio values are given in parenthesis in the table). This shows how significant the JH is which lies outside the nightside auroral zone, e.g. in the cusp, but studying the dayside $\mathrm{JH}$ as such is outside the scope of this paper.

In Fig. 8 we decompose the JH into three MLT sectors (three columns) and for different solar illumination conditions (three rows). Formulas for the fitted solid line are given in each plot, as well as in Table 1, rows 4-6, and estimates of the total JH are given in Table 4, columns 3-5. The JH 
Astrid-2/EMMA JH, All conds

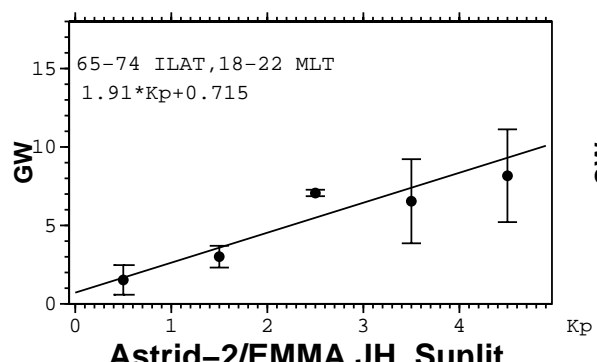

Astrid-2/EMMA JH, Sunlit

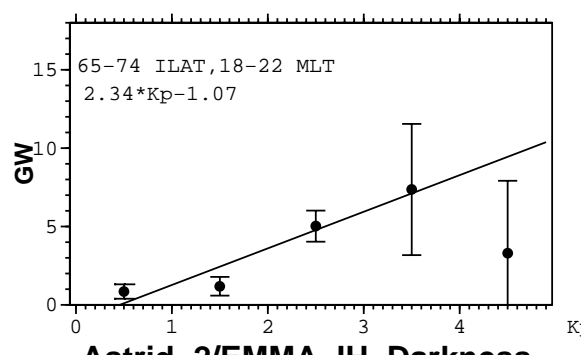

Astrid-2/EMMA JH, Darkness

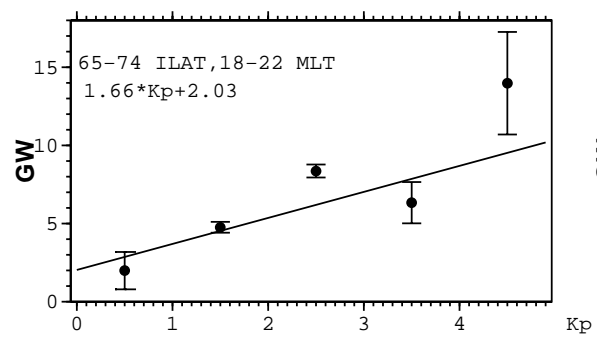

Astrid-2/EMMA JH, All conds

Astrid-2/EMMA JH, All conds

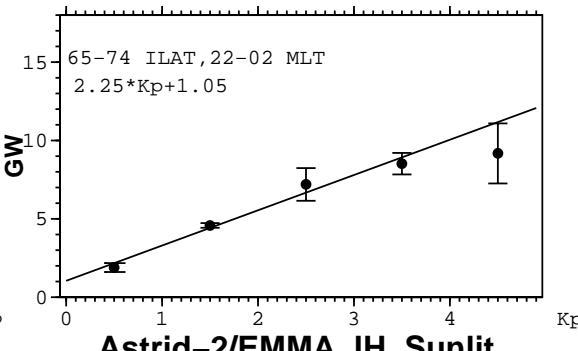

Astrid-2/EMMA JH, Sunlit

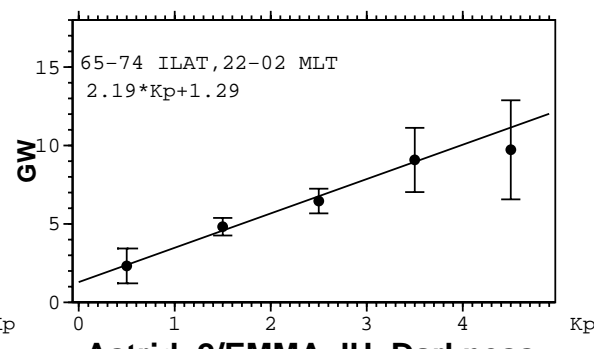

Astrid-2/EMMA JH, Darkness

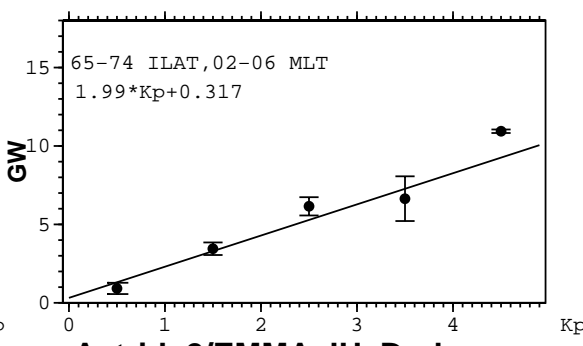

Astrid-2/EMMA JH, Darkness

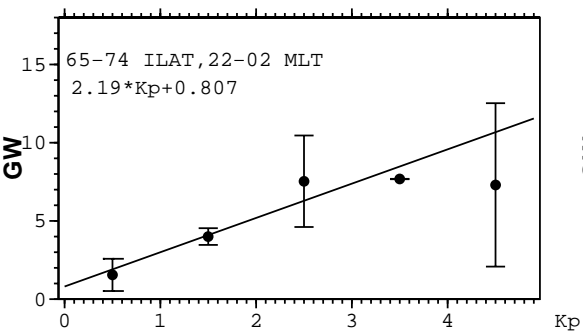

Fig. 8. Dependence of the average Joule heating on $K_{p}$ in the auroral zone (ILAT 65-74) for various MLT and solar illumination conditions. The three columns show the average Joule heating for three different nighttime MLT sectors and the upper row for all solar illumination conditions, the middle row for sunlit conditions and the bottom row for darkness conditions. The solid lines shows a fit to the data and the corresponding formula is shown in each subplot, as well as in Table 1, row 7-9. The Joule heating is roughly the same for sunlit and darkness conditions apart from MLT 18-22, where they are much higher for darkness conditions.

is highest in the midnight MLT sector and smallest in the evening sector. There is not much difference in JH in sunlit and darkness conditions, but the variability is larger in darkness (larger error bars).

\section{Statistical JH and solar wind}

In addition to using the geomagnetic indices $K_{p}$ and $A E$, it is useful for some applications (e.g. for comparison with MHD simulations) to know the average $\mathrm{JH}$ as a function of solar wind parameters directly. Here we consider especially two such parameters, the Akasofu epsilon parameter and the solar wind kinetic energy flux that would be incident on Earth in the absence of a magnetosphere. The epsilon parameter (Akasofu, 1981) is given by

$\epsilon=\frac{4 \pi}{\mu_{0}} v B^{2} L^{2} \sin ^{4}\left(\frac{\theta}{2}\right)$, where $B$ is the magnitude of the interplanetery magnetic field (IMF), $v$ is the solar wind speed, $L=7 R_{E}$ is a length scale of the magnetosphere and $\theta$ is the IMF clock angle $\left(\tan \theta=B_{y} / B_{z}\right)$.

The solar wind kinetic energy flux $W$ incident on one hemisphere of the Earth in the absence of a magnetosphere is defined by

$W=\frac{1}{2}\left(\frac{1}{2} \rho v^{3}\right)\left(\pi R_{E}^{2}\right)$,

where $R_{E}$ is the Earth's radius and the first factor $1 / 2$ comes from considering only one hemisphere. The $W$-parameter has not been traditionally used in discussing magnetospheric energetics, but it has recently been found to correlate rather well with the total ionospheric energy input (JH plus particle precipitation) obtained from an MHD simulation (Palmroth et al., 2004) in cases where the energy transfer variations are dominated by solar wind plasma parameters and not IMF direction changes. It may look surprising that the length scale in the definition of $W$ is the Earth's radius; although the size 
Table 3. Joule heating as a function of epsilon parameter $(\epsilon)$ and hemispheric solar wind power $\left.W=0.5\left((1 / 2) \rho v^{3}\right)\left(\pi R_{E}^{2}\right)\right)$.

\begin{tabular}{lll}
\hline & ILAT 55-90 & ILAT 65-74, MLT 18-06 \\
\hline$\epsilon$ & $0.57 \epsilon+19 \mathrm{GW}$ & $0.15 \epsilon+6.7 \mathrm{GW}$ \\
$W$ & $2.5 W-7 \mathrm{GW}$ & $0.46 W+3 \mathrm{GW}$
\end{tabular}

\section{Astrid-2/EMMA JH, All conds}

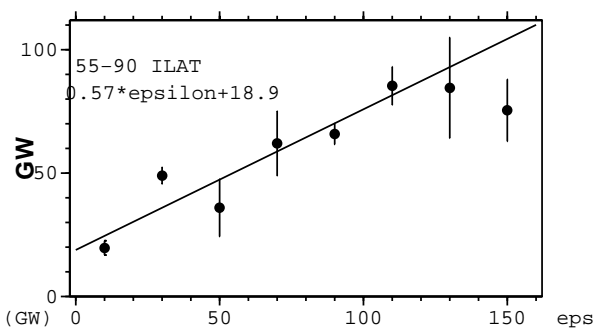

(a)

Astrid-2/EMMA JH, All conds

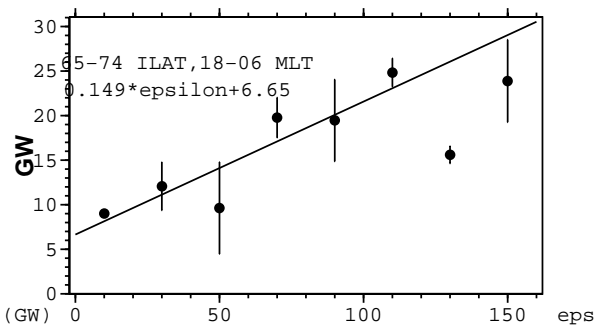

(b)

Fig. 9. The Joule heating depending on the epsilon parameter (Eq. 8) for (a) ILAT 55-90, (b) ILAT 65-74. All MLT and all solar illumination conditions are included. The solid lines corresponds to a fit to the data and the corresponding formula is given in each subplot, as well as in Table 3, row 1. Notice that the JH value is for one hemisphere only, whereas $\epsilon$ is a global parameter; hence, the result that $\mathrm{JH}$ and $\epsilon$ are equal in this plot means that $\epsilon$ underestimates the energy input.

of the planet Earth is probably not significant in determining how much solar wind energy enters the magnetosphere, it is significant in determining the ionospheric energy input because the Earth's "contact area" with its plasma environment is proportional to $R_{E}^{2}$. Using the parameter $W$ it is possible to discuss an interesting question: is the average net effect of the magnetosphere to protect the Earth from the energy flux of the solar wind, or is it rather to act as an "antenna" and amplify the received energy input?

Figure 9 shows the statistical dependence of hemispheric $\mathrm{JH}$ on the epsilon parameter (a) above $55^{\circ}$ ILAT only and (b) in the nightside auroral zone (ILAT 65-74, MLT 18-06). The hemispheric $\mathrm{JH}$ is roughly half of $\epsilon$, i.e. globally $\mathrm{JH}$ and $\epsilon$ are statistically nearly equal. Taken literally, this is consistent with the idea that $\epsilon$ is, indeed, the correct energy input (sum of $\mathrm{JH}$, particle precipitation and ring current energisation) and that $\mathrm{JH}$ is the largest among these three energy

\section{Astrid-2/EMMA JH, All conds}

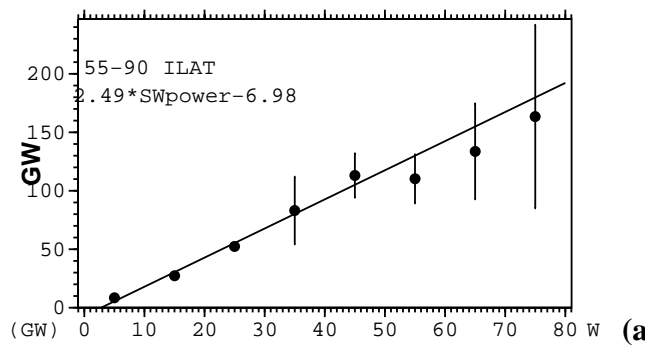

Astrid-2/EMMA JH, All conds

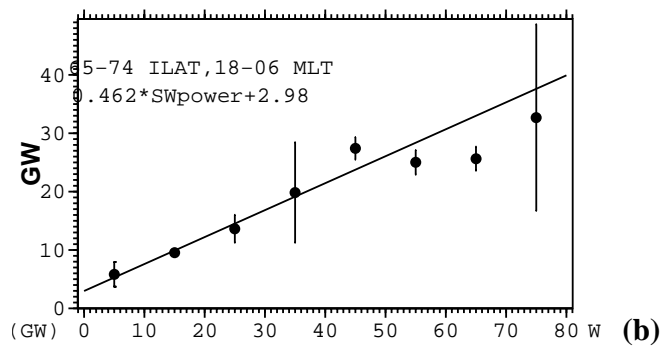

Fig. 10. The Joule heating depending on the solar wind power parameter $W$ (Eq. 9) for (a) ILAT 55-90, (b) ILAT 65-74. All MLT and all solar illumination conditions are included. The solid lines correspond to a fit to the data and the corresponding formula is given in each subplot, as well as in Table 3, row 2. Notice that $W$ is defined for one hemisphere only, as is the $\mathrm{JH}$.

consumption mechanisms. The result is, however, sensitive to the employed definition of $\epsilon$ : had we used the transverse IMF instead of total IMF in its definition (there are theoretical arguments favouring the first choice; Koskinen and Tanskanen, 2002), the average $\epsilon$ would have been nearly two times smaller, ruining the good agreement. It is likely that the agreement is, in any case, fortuitous to some extent.

Similarly, Fig. 10 shows the statistical dependence of hemispheric $\mathrm{JH}$ on the hemispheric solar wind kinetic energy flux $W$ (Eq. 9). The total hemispheric JH is about 2.5 times higher than hemispheric $W$. Since the JH is an underestimate for the total ionospheric energy input, this means that the magnetosphere does not protect the Earth from the energy flux of the solar wind, but rather acts as an "antenna" that magnifies the received power to some (modest) extent.

Notice that our statistical analysis does not give any firm indications of how good or how bad $\epsilon$ or $W$ is in describing the time variations of the energy input: any parameter that depends on the solar wind and IMF can be made to agree statistically with our JH results if scaled suitably. Nevertheless, one notices by comparing the statistical scatter in Figs. 9 and 10 that the scatter for $W$ is smaller than for $\epsilon$. This insinuates that $W$ is able to describe the $K_{p}$ variations of $\mathrm{JH}$ as well as $\epsilon$ or even better. 
Table 4. Total Joule heating in designated region. The first column shows the total global (ILAT 55-90) Joule heating. This can be compared with the total nightside auroral zone Poynting flux (column 2). In parentheses the portion of Joule heating going into the nightside auroral zone relative to the total global Joule heating is shown. In columns 3-5 the total auroral zone related Joule heating is given for each nightside MLT sector.

\begin{tabular}{llllll}
\hline & All MLT & MLT 18-06 & MLT 18-22 & MLT 22-02 & MLT 02-06 \\
\hline & ILAT 55-90 & ILAT 65-74 & ILAT 65-74 & ILAT 65-74 & ILAT 65-74 \\
\hline$K_{p} \leq 2$, All conds & 27.1 & $8.07 \mathrm{GW}(0.30)$ & $2.53 \mathrm{GW}$ & $3.74 \mathrm{GW}$ & $1.80 \mathrm{GW}$ \\
$K_{p} \leq 2$, Sunlit & 32.1 & $7.79 \mathrm{GW}(0.24)$ & $1.17 \mathrm{GW}$ & $4.03 \mathrm{GW}$ & $2.59 \mathrm{GW}$ \\
$K_{p} \leq 2$, Darkness & 14.4 & $7.00 \mathrm{GW}(0.49)$ & $3.75 \mathrm{GW}$ & $3.20 \mathrm{GW}$ & $0.05 \mathrm{GW}$ \\
\hline$K_{p}>2$, All conds & 114 & $25.3 \mathrm{GW}(0.22)$ & $7.61 \mathrm{GW}$ & $8.94 \mathrm{GW}$ & $8.76 \mathrm{GW}$ \\
$K_{p}>2$, Sunlit & 115 & $24.4 \mathrm{GW}(0.21)$ & $6.98 \mathrm{GW}$ & $8.59 \mathrm{GW}$ & $8.85 \mathrm{GW}$ \\
$K_{p}>2$, Darkness & 92 & $26.5 \mathrm{GW}(0.29)$ & $8.94 \mathrm{GW}$ & $8.86 \mathrm{GW}$ & $8.68 \mathrm{GW}$ \\
\hline
\end{tabular}

\section{Summary}

We briefly summarise our findings.

1. Our JH results agree with Foster et al. (1983) rather well (Fig. 5a). This is significant, as the studies used quite different satellite-based methods and also the electric field instrument type was different. Furthermore, the difference that remains may be partly explainable by the fact that our study is during solar maximum and Foster's study was during solar minimum. Our results for $\mathrm{JH}$ are slightly higher than those of Ahn et al. (1983).

2. We tabulated formulas for $\mathrm{JH}$ dependence on $K_{p}, A E, \epsilon$ and $W$ for different solar illumination conditions, global and auroral zone ILAT ranges and different nightside MLT sectors (Tables 1-3).

3. The nominal nightside (MLT 18-06) auroral oval (6574 ILAT) receives about $1 / 3$ of the total $\mathrm{JH}$ above 55 ILAT (Table 4). For $K_{p}>2$ the ratio is closer to $1 / 4$, probably because some auroral substorm electric fields then penetrate below $65^{\circ}$ ILAT.

4. In the nightside nominal auroral zone (18-06 MLT, 6574 ILAT), the JH is $\sim 3$ times larger for $K_{p}>2$ than for $K_{p} \leq 2$.

5. When data are binned with respect to $\epsilon$, they are consistent (perhaps fortuitously) with the idea that JH dominates over other energy consumption channels (particle precipitation and ring current) and that the traditional scale parameter $\left(7 R_{E}\right)$ in the definition of $\epsilon$ is statistically nearly correct. The data would also be consistent with the interpretation that particle precipitation and/or ring current are/is important as well and that $\epsilon$ underestimates the total energy transfer to some extent.

6. The global $\mathrm{JH}$ is on the average about 2.5 times higher than the solar wind kinetic energy flux that would be incident on Earth in the absence of a magnetosphere. Thus, the magnetosphere does not protect the Earth from the energy flux of the solar wind, but rather acts as an "antenna" that magnifies the energy input to a modest extent.

\section{Discussion}

\subsection{Comparison with previous statistical studies}

This study is the first time ionospheric $\mathrm{JH}$ is studied statistically using low-altitude double-probe electric field measurements. Previous studies using the Poynting flux method (Gary et al., 1994, 1995) or the $\Sigma_{P} E^{2}$ method (Foster et al., 1983) used drift-meter type electric field instruments. The time resolution of this study $(1 \mathrm{~s})$ is higher than that $(15 \mathrm{~s})$ used by Foster et al. (1983). In terms of size of the database (3886 auroral crossings), our study falls in between the 25000 orbits of Foster et al. (1983) and the 576 orbits of Gary et al. (1995). This study contains a comprehensive set of fitted formulas (Tables 1-3) where the JH in some ILAT and MLT region is expressed as a function of $K_{p}, A E \epsilon$ and $W$ parameters (Eq. 9).

Despite some differences in details, generally our results are in rather good agreement with Foster et al. (1983) and also those of Ahn et al. (1983), although our JH levels are somewhat higher than those found earlier.

Our presentation of statistical JH results was divided into two parts: global JH above 55 ILAT and JH in 18-06 MLT, 65-74 ILAT. The latter is intended to serve future studies of energy budget in the nightside auroral region.

\subsection{Comparison with global MHD simulation}

As we pointed out in the Introduction, the GUMICS-4 MHD simulation (Janhunen, 1996) produces JH levels that are smaller than the statistical results obtained here and elsewhere (Palmroth et al., 2004) and that any explanation attempt of this discrepency must take into account the fact that the GUMICS-4 cross-polar potential drop is nevertheless in agreement with SuperDARN radar results (Janhunen et al., 
1998). The fact that some other global MHD models produce larger JH levels than GUMICS-4 while in turn overestimating the cross-polar potential drop underlines the physical nature of the problem.

From the inspection of GUMICS-4 and Astrid-2 results, we are inclined to think that at least part of the explanation of the dilemma lies in the fact that GUMICS-4 has rather weak Region-2 field-aligned currents (FACs), probably due to insufficient plasma pressure and ring currents in the inner magnetosphere, and, consequently, the Region- 1 currents in the simulation mainly close among themselves and not with the neighbouring Region-2 current sheet. In contrast, significant JH in Astrid-2 data is often associated with the area between the Region- 1 and Region- 2 current sheets in the auroral oval. The cross-polar potential drop mainly depends on the net Region-1 minus Region-2 current. By increasing both Region- 1 and Region- 2 by the same amount, the JH between them increases, but the cross-polar potential drop remains almost unchanged.

\subsection{General discussion}

This paper contains improved and more detailed estimates than have been possible to obtain before of the statistical ionospheric Joule heating as a function of many different parameters. The results should give useful building blocks for future quantitative studies of a global magnetospheric power budget, as well as the power budget in the nightside auroral region. What still remains a related and open question is how the Joule heating depends on space and time and on the solar wind. We hope that global MHD simulations eventually will shed light on this question, provided that they can first be brought into correspondence with the observational statistical Joule heating.

Acknowledgements. The work of AO was supported by the Swedish Research Council and that of PJ by the Academy of Finland. The authors acknowledge A. Vaivads for useful discussions concerning the Poynting flux. The Astrid-2 satellite project was mainly funded by the Swedish National Space Board.

The Editor in Chief thanks a referee for his/her help in evaluating this paper.

\section{References}

Ahn, B.-H., Akasofu, A.-I., and Kamide, Y.: The joule heat production rate and the particle energy injection rate as a function of the geomagnetic indices AE and AL, J. Geophys. Res., 88, 6275-6287, 1983.

Akasofu, S.-I.: Energy coupling between the solar wind and the magnetosphere, Space Sci. Rev., 28, 121-190, 1981.
Blomberg, L. G., Marklund, G. T., Lindqvist, P.-A., Primdahl, F., Brauer, P., Bylander, L., Cumnock, J. A., Eriksson, S., Ivchenko, N., Karlsson, T., Kullen, A., J. M. Merayo, G., Pedersen, E. B., and Petersen, J. R.: EMMA - the electric and magnetic monitor of the aurora on Astrid-2, Ann. Geophys., 22, 115-123, 2004.

Eriksson, S., Blomberg, L. G., Ivchenko, N., Karlsson, T., and Marklund, G. T.: Magnetospheric response to the solar wind as indicated by the cross-polar potentila drop and the lowlatitude asymmetric disturbance field, Ann. Geophys., 19, 649653, 2001.

Foster, J. C., St.-Maurice, J.-P., and Abreu, V. J.: Joule heating at high latitudes, J. Geophys. Res., 88, 4885-4896, 1983.

Gary, J. B., Heelis, R. A., Hanson, W. B., and Slavin, J. A.: Fieldaligned Poynting flux observation in the high latitude ionosphere, J. Geophys. Res., 99, 11 417-11 427, 1994.

Gary, J. B., Heelis, R. A., and Thayer, J. P.: Summary of fieldaligned Poynting flux observations from DE 2, Geophys. Res. Lett., 22, 1861-1864, 1995.

Ivchenko, N., Facciolo, L., Lindqvist, P.-A., Kekkonen, P., and Holback, B.: Disturbance of plasma environment in the vicinity of the Astrid-2 microsatellite, Ann. Geophys., 19, 655-666, 2001.

Jackson, J. D.: Classical electrodynamics, 3rd ed., Wiley, New York, 1999.

Janhunen, P.: GUMICS-3 - a global ionosphere-magnetosphere coupling simulation with high ionospheric resolution, in: Proc. Environmental modelling for space-based applications, 18-20 Sept. 1996, ESTEC, The Netherlands, ESA SP-392, 1996.

Janhunen, P., Amm, O., and Greenwald, R. A.: Adaptive mesh in global magnetosphere simulation: Comparison with global convection, Proceedings of the 1998 Cambridge Symposium/Workshop in Geoplasma Physics on 'Multiscale Phenomena in Space Plasmas II', Physics of Space Plasmas 15, edited by: Chang, T. and Jasperse, J. R., MIT, 1998.

Kelley, M. C., Knudsen, D. J., and Vickrey, J. F.: Poyntingflux measurements on a satellite: A diagnostic tool for space research, J. Geophys. Res., 96, 201-207, 1991.

Koskinen, H. E. J. and Tanskanen, E. J.: Magnetospheric energy budget and the epsilon parameter, J. Geophys. Res., 107, A11, 1415, doi:10.1029/2002JA009283, SMP 42-1, 2002.

Marklund, G. T., Blomberg, L. G., and Persson, S.: Astrid-2, an advanced microsatellite for auroral research, Ann. Geophys., 19, 589-592, 2001.

Palmroth, M., Janhunen, P., Pulkkinen, T. I., and Koskinen, H. E. J.: Ionospheric energy input as a function of solar wind parameters: global MHD simulation results, Ann. Geophys., 22, 549-566, 2004.

Richmond, D. A.: Ionospheric electrodynamics, Handbook of Atmospheric Electrodynamics, vol. 2, edited by: Volland, H., 249290, CRC Press, Boca Raton, Fla., 1995.

Tanskanen, E., Pulkkinen, T. I., Koskinen, H. E. J., and Slavin, J. A.: Substorm eneryg budget during low and high solar activity: 1997 and 1999 compared, J. Geophys. Res., 107, A6, 1086, doi:10.1029/2001JA900153, SMP 15-1, 2002.

Vickrey, J. F., Vondrak, R. R., and Matthews, S. J.: Energy deposition by precipitating particles and Joule dissipation in the auroral ionosphere, J. Geophys. Res., 87, 5184-5196, 1982. 